\title{
Case study on how to develop 3D labs with theoretical, experimental, and computational goals
}

\author{
Ashley R. Carter ${ }^{1}$ \\ ${ }^{1}$ Physics Department, Amherst College, Merrill Science Center, Amherst, MA, 01002
}

\begin{abstract}
Overhauling a laboratory experiment, course, or curriculum is a daunting process. Here, I describe a fourstep process our department used to overhaul our laboratory curriculum and courses. This four-step process includes: 1) identifying learning goals, 2) describing current practices, 3) making changes, and 4) planning for assessment. In addition, I describe how we updated experiments in the courses to be "3D". These "3D" experiments are designed to meet three different types of goals: theoretical goals, experimental goals, and computational goals.
\end{abstract}

\section{INTRODUCTION}

There is no better time to overhaul physics laboratory courses than right now. Recently, research has shown that laboratories that have goals of reinforcing course content have no value added when it comes to learning that content for the final exam [1]. Given the expense and effort required to mount a laboratory course, it is worthwhile to take the time to rethink courses with these goals. After all, the laboratory is more than just a vehicle for course content; it is the method by which we teach future scientists to do science.

It is also a good time to update laboratory courses as the American Association of Physics Teachers (AAPT) has just published a new set of laboratory guidelines, which include learning outcomes in six focus areas: constructing knowledge, modeling, designing experiments, developing technical and practical laboratory skills, analyzing and visualizing data, and communicating physics [2]. Laboratory courses that want to implement best practices should be updated to meet these learning goals.

In addition, we need "3D laboratory courses" that integrate theoretical, experimental, and computational goals. That is, laboratory courses should not just cover the theoretical topics or just meet the experimental goals outlined in the AAPT laboratory guidelines. Instead, the laboratory should provide authentic experiences that mix theoretical, experimental, and computational learning outcomes. As the AAPT has just published computational guidelines that outline goals for how to construct knowledge using computational tools, computational physics skills, and computational technical skills [3], the time is ripe to update the laboratory.

However, updating the laboratory is an incredibly daunting task. Where should we start? What should we do?

Here I provide a roadmap that our department has used to update the laboratory for 3D physics. For updating the curriculum and the courses in the curriculum, we used a four-step process: 1) identifying learning goals, 2) describing current practices, 3) making changes, and 4) planning for assessment. This process is based on other work [4-8] and modified to suit our particular needs. To create 3D experiments, we modified existing research training modules to create experiment manuals based on the 3D goals rather than the physics concepts. Using these processes, we are developing 3D labs with theoretical, experimental, and computational goals.

\section{FOUR STEP PROCESS FOR UPDATING CURRICULUM OR COURSE}

\section{A. Identifying learning goals}

Our first step in updating the laboratory curriculum or course started with identifying the learning goals for students. The learning goals are the things that you want students to be able to do or know at the end of the course. The reason for starting with these goals is twofold. First, when designing the curriculum or course, you need these goals to guide the changes you will make. Second, most instructors will be able to agree on the goals [6], making it a great place to start.

To identify curricular goals for our laboratory sequence, we held one meeting where all interested parties could attend. Before the meeting, everyone was given a copy of the AAPT laboratory guidelines and the AAPT computational guidelines. This facilitated the discussion during the meeting as almost everyone agreed on the goals in these documents. In addition, the purpose for the meeting - to update the laboratory sequence to meet theoretical, experimental, and computational goals - was clearly outlined. This purpose allowed our group to stay focused on these three types of goals and complete the task in just one hour. Finally, during the meeting we tried to keep the goals broad, rather than specific. This allowed us to list all of the goals easily without too much discussion. For a list of the goals we came up with see the "Goals and Practices BLANK" document [9].

To identify goals for each course, we used the same strategy of gathering for a one hour meeting. We found that there was almost perfect overlap between our course goals and our curricular goals. This overlap is most likely because the goals in the AAPT laboratory guidelines and the AAPT computational guidelines documents are so broad. For example, one of the goals is to "analyze and display data using statistical methods" [2]. This is definitely a goal for the curriculum and for each of our four courses in our laboratory sequence. However, the degree of sophistication in each of the courses is different. Analyzing and displaying data in an introductory lab course may just involve plotting a line, while in an advanced course it may involve plotting a Poisson distribution and the residuals. Thus, the goals for each course are the same, but the actual practices in the different courses are different.

Our second step in updating the laboratory curriculum or 
course was to describe the current practice in the department. This was an easy step since we just needed to recall what happened in the course or courses the last time they were taught. This step not only moves things along, but also increases agreement among the group.

We accomplished this task in a one hour meeting by creating a single document that described the practices in our department, see the "Goals and Practices EXAMPLE" document [9]. In this document, we listed the four courses in our laboratory sequence along the top. Then, we placed the goals for the course along the side, creating a grid. At each location in the grid, we listed the practice in that course for meeting the goal. For example, if the goal was for students to be able "to process and represent data" using technical computing skills [3], then in the introductory lab we wrote that our current practice is to tell students exactly how to process and represent the data in the laboratory directions. In contrast, the current practice in the advanced lab is to have students decide what data to take, how to process it, and what representation to choose. Each one of these practices is added to the grid at the correct location. To make the document more useful, we grouped the goals into the three categories of theoretical, experimental, and computational. This grid allowed us to see the current practices in each course at a glance.

\section{B. Making changes}

Our third step of the process was to assess our goals and current practices and decide on changes. This involved another one hour meeting of the group.

First, we looked at the empty grid squares in our "Goals and Practices" document. An empty grid square means that there are no current practices in the course that help students meet that goal. Sometimes this is by design. For example, troubleshooting is an experimental goal for our courses, yet in the introductory lab we keep troubleshooting to a minimum due to time constraints. Other times, the grid squares are empty because we have not been meeting our goals, or we have identified a new goal for the course. In these cases, we filled the empty grid squares with practices that we would like to implement.

Second, we looked at adding practices. Sometimes there were not enough practices to meet a particular goal. For example, in the computational goals section in "use a mathematical/computational model to represent reality" we had two courses that gave students the model and then asked them to verify the model with their data. In a third course, we had the students come up with a model and refine it. To bridge these courses, we added practices to the introductory lab of giving them part of the model or walking them through how to think of models. Adding these practices allowed us to scaffold the students from one course to the next.

Third, when adding practices or even describing current practices we made sure to note alternatives. There are many ways to achieve a particular goal and one method might work well for one instructor and not for another. To be inclusive of these alternate methods, we wrote them all down. For example, there were many different practices that instructors wanted to implement to improve student communication skills. Our traditional method is to require three written lab reports. However, some instructors wanted to try scaffolded reports [10], oral presentations, or executive summaries. Rather than choosing one method, we just list them all as alternatives.

Fourth, we did not remove practices; we only added practices and listed alternatives. This document is supposed to represent current practice in the department. If next year, the practices have changed because faculty have updated their practices, then the document will shift naturally. This allows instructors to make changes at their own pace.

\section{Planning for assessment}

The last step in our process was to plan for assessment of the curriculum or course. Assessment is necessary [7] so that we know how to update the course.

While it is possible to use nationally recognized surveys [7] or practical evaluations [11] as laboratory assessment tools, our current practice is to use course evaluations, see the "Course evaluation" document for more details [9]. In the course evaluation, students rank their progress at achieving each goal, allowing us to continuously update the laboratory.

\section{CREATING 3D EXPERIMENTS}

Once the course and curriculum have been updated, the next step is to design 3D experiments. But, what experiments should be used? And, how will departments continuously update these experiments to meet the needs identified in the assessment of the course?

\section{A. Need for plug and play experiments}

One traditional method for updating experiments is for the instructor of the course to rewrite and rework all the experiments in the lab each year. Given the time it takes to update experiments, this policy usually means that the laboratory is rarely updated.

Another method is for the department to identify a "task force" of two or more people to update or create experiments. This method typically works to update the lab, but the problem is that a task force is rarely created.

Finally, another method is to have a national repository of experiments that instructors can use to update their courses. Currently, instructors can look to physics education companies, the AAPT ComPADRE website, or to physics education journals for experiments. But, experiments in these reposito- 
ries are not uniform and require a lot of work on the part of the instructor to outfit the experiment for their institution.

What is needed is a repository of experiments that are "plug and play". "Plug" here means that the experiment can be plugged into a wide range of courses. This means that the experiment must be stand-alone and it must be rich enough to cover an array of theoretical, experimental, and computational goals. "Play" here means that the experiment can be played during a single three hour time slot or over multiple weeks by different instructors who might want to emphasize different goals. This means that the experiment needs to be written in a way that allows the instructor to play the lab without too much trouble. Instructors can then choose from a set of these plug and play experiments to build a course.

\section{B. Identifying "pluggable" experiments}

For an experiment to be plugged into a course it needs to be rich enough to meet a large variety of theoretical, experimental, and computational goals. Right now the experiments that meet this criteria are often research experiments. This is because research experiments are authentic representations of modern science that cover multiple goals. However, these research experiments are often too time-consuming and difficult to fit into the required lab period and require savvy instructors to think of doable projects.

Here, I propose that instead of using a research experience as the lab, a better solution might be to create $3 \mathrm{D}$ experiments out of "training exercises" found in research labs. Training exercises are typically rich enough that they cover all of the theoretical, experimental, and computational goals. Yet, training exercises are the doable version of a research experience that students can access and have ownership over.

For example, many biophysics research laboratories track molecules undergoing Brownian motion, including molecular motors, DNA remodelers, and cytoskeletal filaments. To transition students into these research groups, there is often a first training exercise where students track the Brownian motion of micron-sized particles in water [12], making this a great choice for a laboratory experiment. Another training exercise for a biophysics course might be using an atomic force microscope (AFM) to visualize single DNA molecules on a surface [13]. Finally, a common training exercise for research in optics or atomic physics is to align a laser to a path set by two irises. Often this is followed by adding a series of lenses to set up a beam expander. All of these training exercises are good choices for student experiments in the classroom because they are simple enough that the students can have ownership over the experiment and yet rich enough that they allow for the experiment to be plugged into many different courses.

Of course, another solution is to upgrade the experiments that are typically taught in the laboratory to have $3 \mathrm{D}$ goals. Physics education companies could take the lead here.

\section{Writing "playable" experiments}

It is not enough to identify experiments with $3 \mathrm{D}$ goals. We must also write the manuals for these experiments so that instructors can easily "play" the experiment. Currently, manuals are organized by physics concept: measure this physical quantity, observe this physical quantity, answer a question about this physical quantity. However, if we want to emphasize theoretical, experimental, and computational skills, then the experiment manual needs to be reorganized by goals: model this, design this, use this technical skill.

Furthermore, in order for the experiment to be "playable" for multiple instructors, then the manual needs to list options. Multiple options allow instructors to choose different paths. For example, three options for the prelab for the Brownian motion experiment could be: 1) use a random number generator to simulate 10 tracks (goal = computational physics skills), 2) calculate a mean-squared-displacement by hand for a set of given $x-y$ data (goal = experimental data analysis), or 3 ) think about ways to remove fluid flow from samples (goal $=$ experimental technical skills). For more examples, see the "Waves Laboratory Manual" [9]. Writing manuals with options based on the 3D goals, creates plug and play experiments.

\section{CONCLUSIONS}

Here we describe a method for updating laboratory experiments, courses, and curriculums. For courses and curriculums we used a four-step process of 1) identifying learning goals, 2) describing current practices, 3) making changes, and 4) planning for assessment. This process was useful for our department because it was easy to implement (four 1-hour meetings) and did not create disagreement in the department. During the first meeting, we used the AAPT documents as a reference to select learning goals. In the second and third meetings, we noted our current practices and what practices or changes we would add. Finally, in the fourth meeting, we met with the school's instructional designer to update our course evaluations to plan for assessment. Moving forward, individual instructors will use the assessments to update their courses, and the department will meet yearly to reevaluate.

In addition, we also describe a path for updating an experiment so that it is "plug and play". An experiment that can be "plugged" into multiple courses will address multiple theoretical, experimental, and computational goals. An experiment that can be "played" in multiple courses would display those goals in a lab manual that allows instructors to choose between the goals for different sections of the lab. This arrangement of the lab manual allows for easy updates based on the assessments. In this way, our department is moving forward to create laboratory experiments, courses, and curriculums that will teach the theoretical, experimental, and computational skills necessary for doing experimental physics. 


\section{ACKNOWLEDGMENTS}

This work was supported by the Research Corporation
(ARC, Award \#23239), an NSF CAREER award (ARC, Project \#1653501), and Amherst College.
[1] N. G. Holmes et al., Phys. Rev. PER 13, 1010129 (2017).

[2] J. Kozminski et al., AAPT Recommendations for the Undergraduate Physics Laboratory Curriculum (2014).

[3] E. Behringer et al., AAPT Recommendations for Computational Physics in the Undergraduate Physics Curriculum (2016).

[4] M. D. Caballero and S. J. Pollock, Am. J. Phys. 82, (3) 231-237 (2014).

[5] S. V. Chasteen and S. J. Pollock, AIP Conf. Proc. 1064, (1) 91-94 (2008).
[6] R. E. Pepper et al., AIP Conf. Proc. 1413, 1 291-294 (2012).

[7] B. M. Zwickl et al., Am. J. Phys. 81, (1) 63-70 (2013).

[8] B. M. Zwickl et al., Am. J. Phys. 82, (9) 876-882 (2014).

[9] http://www3.amherst.edu/ acarter/pages/teaching.html. Retrieved 7/10/2018.

[10] L. J. Deiner et al., J. Chem. Ed. 89, (12) 1511-1514 (2012).

[11] M. Finegold and J. Meyer, Stud. Educ. Eval. 11, (3) 321-326 (1985).

[12] M. A. Catipovic et al., Am. J. Phys. 81, (7) 485-491 (2013).

[13] L. M. Devenica et al., Am. J. Phys. 84, (4) 301-310 (2016). 